\title{
Greener Synthesis of an Amide by Direct Reaction of an Acid and Amine under Catalytic Conditions
}

\author{
Gabriel Arce, Gonzalo Carrau, Ana Bellomo, David Gonzalez* \\ Depto. de Química Orgánica, Facultad de Química, Universidad de la República (UdelaR), Montevideo, Uruguay \\ *Corresponding author: davidg@fq.edu.uy
}

Received February 13, 2015; Revised March 03, 2015; Accepted March 10, 2015

\begin{abstract}
A simple and high-yielding laboratory experiment that involves the boric acid catalyzed amidation of benzoic acid is described. The experiment makes use of recent chemistry, it is appropriate for advanced undergraduate chemistry students, and demonstrates several important concepts including homogeneous catalysis and green chemistry.
\end{abstract}

Keywords: Upper-Division Undergraduate, organic chemistry, green chemistry, catalysis, amides

Cite This Article: Gabriel Arce, Gonzalo Carrau, Ana Bellomo, and David Gonzalez, "Greener Synthesis of an Amide by Direct Reaction of an Acid and Amine under Catalytic Conditions." World Journal of Chemical Education vol. 3, no. 1 (2015): 27-29. doi: 10.12691/wjce-3-1-4.

\section{Introduction}

The emergence of green chemistry in the nineties [1] asked the question of whether the traditional focus of organic synthesis on yield and selectivity had overlooked the environmental concern associated with many synthetic reactions.

The importance of the peptide bond for the pharmaceutical industry [2,3] and the increasing costs of waste disposal have motivated the research for new methods for amide bond formation avoiding the generation of undesired materials and increasing atom economy. A common teaching paradigm in junior organic chemistry states that you cannot prepare an amide from the reaction of a carboxylic acid and an amine because the acid-base chemistry will preclude the nucleophilic attack on the carboxylic acid. Therefore, non-acidic derivatives such as acid chlorides and anhydrides should be prepared before attempting the reaction with the amine. The argument is strong and works well to teach reactivity, nucleophilic attack, and acid-base chemistry. Actually, it is correct that an acid and an amine will form a salt, but you do not necessarily need to synthesize potentially hazardous chemicals such as acid chlorides in order to promote an amide bond formation. Alternative ways to activate carboxylic acids are well known. Coupling mediated by carbodiimides such as DCC or EDC work in excellent yield and in fact is the foundation of automatic peptide synthesis [4,5]. Carbodiimides are not alone in the plethora of carboxylic acid activators, 1-hydroxybenzotriazole (HOBt) and $N$-acylimidazoles are other reagents frequently used in the synthesis of amides [6]. However, all these approaches are atom uneconomical and the large entities used to activate the acid translate into very significant amounts of waste.
Recently, there have been many reports on the direct amidation of carboxylic acids. Among the techniques described are microwave activation [7,8], biocatalytic reactions $[9,10,11]$ and catalytic boric acid activation of the carboxylic acid. The latter approach is particularly attractive since it is operationally simple, easily scalable and very high yielding (Figure 1). The methodology was first developed at Emisphere Technologies, Inc. resulting in a patent [12] and later it was fully disclosed and described in depth in an Organic Synthesis procedure [13]. Several other variations and additions are still being developed as exemplified by several publications in the last five years $[14,15]$.

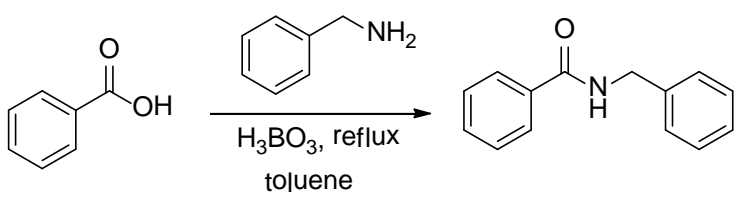

Figure 1. Boric acid catalyzed amidation reaction

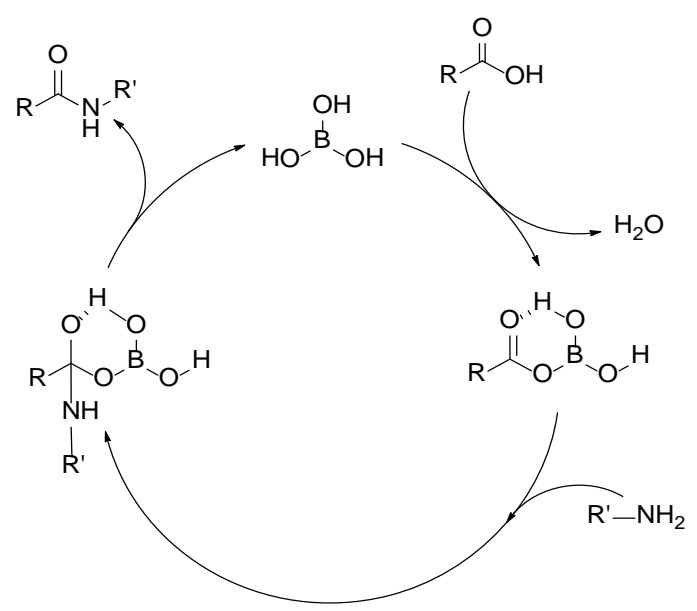

Figure 2. Catalytic cycle for the boric acid catalyzed amidation reaction 
It is proposed that the boric acid reacts with the carboxylic acid to form a mixed anhydride as the actual acylating agent [13]. Upon reaction with the amine, this intermediate forms the desired carboxamide and regenerates the catalytically active boric acid (Figure 2).

During discussion of the mechanism with the students it quickly became evident for them that the amidation is not the direct result of the reaction of the amine and the carboxylic acid. The actual reacting species is an activated form of the acid but unlike traditional methods the derivative is formed in situ and notably only a catalytic amount of mixed anhydride is present at any time during the reaction.

\section{Results and Discussion}

Two advanced undergraduates who were performing an internship in our laboratory tested several aromatic acids. Interestingly, the affordable and less toxic unsubstituted benzoic acid reacted in a superior or similar fashion than other substituted analogs and therefore was chosen for the experiment.

In order to determine the optimum reaction time to obtain a good yield of product without compromising the catalytic character of the reaction we performed several runs to test the influence of the catalyst mass in the reaction rate. Table 1 summarizes the main observed results.

Table 1. Boric acid amidation performed under different reaction conditions

\begin{tabular}{cccc}
\hline entry & $\mathrm{H}_{3} \mathrm{BO}_{3}(\mathrm{~mol} \%)$ & yield & time $(\mathrm{h})$ \\
\hline 1 & 1 & 89 & 20 \\
2 & 10 & 84 & 15 \\
3 & 25 & 80 & 8 \\
4 & 50 & 62 & 5 \\
\hline We first tested the reaction of & benzoic acid and
\end{tabular}
benzylamine with $1 \mathrm{~mol} \%$ of boric acid (entry 1 , Table 1 ) and obtained the amide product in $89 \%$ yield (before crystallization) after $20 \mathrm{~h}$ of reaction. As we were looking for a reaction amenable for a laboratory class, we needed shorter reaction times without sacrificing the good yield obtained. Therefore, we reasoned that increasing the amount of catalyst would allow the reaction to be completed in a shorter time. In that sense, we performed the amidation using 10, 25 and $50 \mathrm{~mol} \%$ of boric acid (entries 2-4, Table 1) and observed that the reaction time can be shortened to $8 \mathrm{~h}$ without significant drop in the molar yield. The reaction can be faster if a $50 \mathrm{~mol} \%$ amount of catalyst is used but the yield is lower, and the use of $50 \mathrm{~mol} \%$ of catalyst opposes the green distinctiveness of the reaction. On the other hand, longer reaction times lead to a larger energy usage while boric acid can be recovered and reused and therefore, utilizing a $50 \mathrm{~mol} \%$ amount of the catalyst could be justified. Recovery of the boric acid is also energy costly and therefore a cautious analysis should be performed before selecting the desirable amount of catalyst to be used. We found interesting to discuss with the students these parameters and how the overall reaction conditions must be assessed before making a decision on a production process.

\section{Conclusion}

This experiment describes a clean reaction, with a relatively simple setup that can be run in a low budget academic environment. Several teaching concepts are taught with this experiment: acid-base vs. nucleophilicity, equilibrium shift, catalyzed reactions, catalytic cycles, and green chemistry. In addition the experiment teaches reaction setup, anhydrous conditions, and Dean-Stark promoted reactions.

This experiment cannot be run in a short lab period since the minimum reaction time (using catalytic amounts of boric acid) is 5 hours. On the other hand, it is ideally incorporated as a short research project for advanced undergraduates. Our students tested different reaction conditions (stoichiometry, heating method, water abstraction techniques, etc). The experience was rewarding and exposed the students to the systematic study of a reaction. It proved to be an excellent opportunity to discuss chemistry concepts and green chemistry with third year chemistry and pharmacy majors.

\section{Experimental Part}

\subsection{Apparatus}

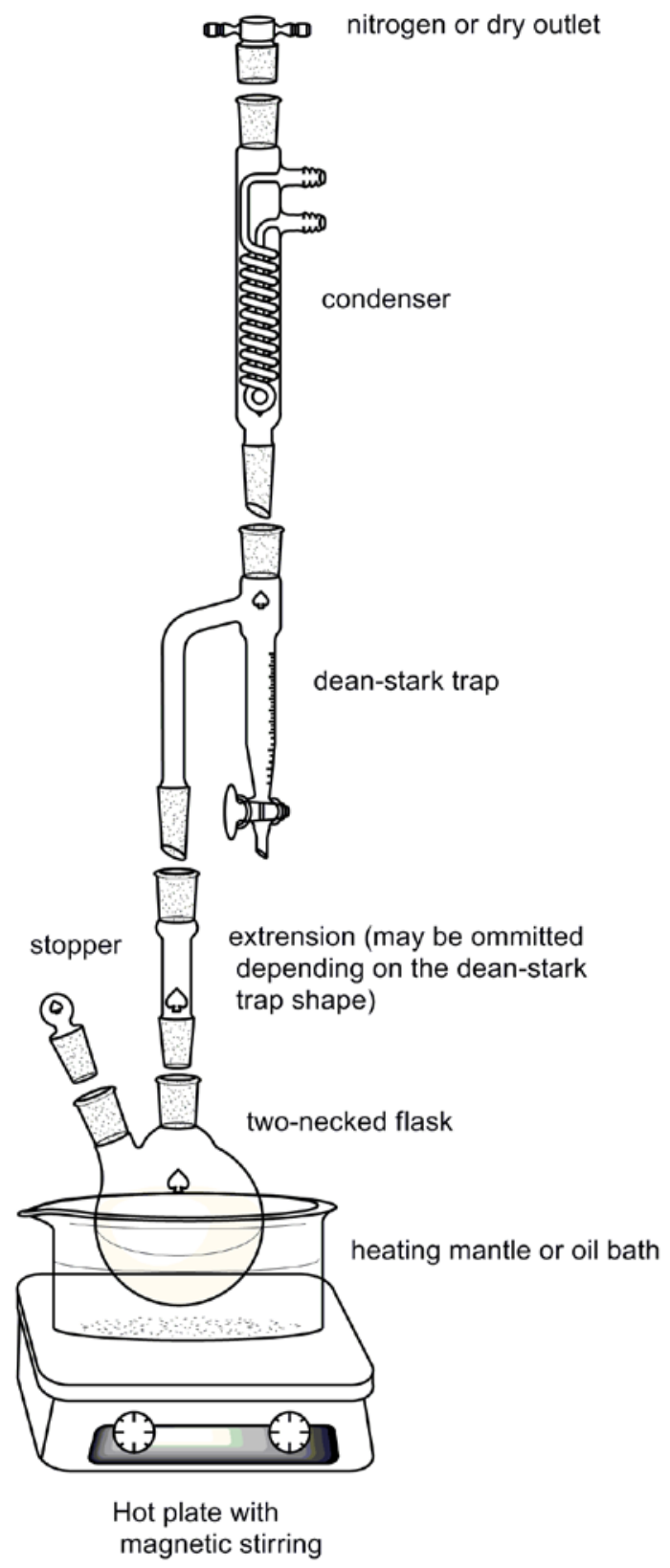




\subsection{Experimental Procedure}

A 250-mL, two-necked, round-bottomed flask was equipped with a magnetic stirring bar and a Dean-Stark trap topped with a reflux condenser with a $\mathrm{CaCl} 2$ moisture trap. Benzoic acid (3.66 g, $0.03 \mathrm{~mol}$ ), boric acid (0.02$1.00 \mathrm{~g}, 1-50 \% \mathrm{~mol})$ and toluene $(88 \mathrm{~mL})$ were added to the reaction vessel. The colorless and slightly turbid reaction mixture was stirred for 10 minutes. After that benzylamine ( $3.4 \mathrm{~mL}, 0.031 \mathrm{~mol}$ ) was added and heating was started by means of an oil bath. The reaction mixture was heated at reflux for $5-20 \mathrm{~h}$ until $0.5 \mathrm{~mL}$ of water were collected in the Dean-Stark trap. The reaction was monitored by TLC $(\mathrm{Rf}=0.5$ (amide); $\mathrm{Rf}=0.7$ (carboxylic acid); hexanes/ethyl acetate, 7/3). The mixture was allowed to cool to room temperature and was poured into $100 \mathrm{~mL}$ of hexanes. The precipitate was collected by suction filtration and the amorphous solid was washed with two $60 \mathrm{~mL}$ portions of water to remove residual boric acid. The resulting solid was dried (3.93-5.64 g, 62-89\%), dissolved in $\mathrm{CDCl}_{3}$, and analyzed by NMR. No signals corresponding to protons of the starting materials were detected in this sample [16,17].

\section{Acknowledgement}

Facultad de Química-UdelaR, PEDECIBA and ANII provided support for this project.

\section{References}

[1] Anastas, P.T. and Warner, J.C., Green Chemistry: Theory and Practice, Oxford University Press, 1998.

[2] Ghose, A.K., Viswanadhan, V.N. and Wendoloski, J.J., J. Comb. Chem., 1. 55-68. 1999.

[3] Walsh, G., Pharmaceutical Biotechnology: Concepts and Applications, Wiley-Default Press, 2007.

[4] Mikolajczyk, S.J. and Kielbasinski, D., Tetrahedron, 37. 233-284. 1981.

[5] Montalbetti, C.A.G.N. and Falque, V., Tetrahedron Lett., 61. 10827-10852. 2005.

[6] Windridge, G.C. and Jorgensen, E.C., J. Am. Chem. Soc., 17. 6318-6319. 1971.

[7] Kappe, C.O., Angew. Chem., Int. Ed., 43. 6250-6284.2004.

[8] Peneux, L., Loupy, A. and Volatron, F., Tetrahedron, 58. 21552162. 2002.

[9] Moree, W.J., Sears, P., Kawashiro, K., Witte, K. and Wong, C.H., J. Am. Chem. Soc., 119. 3942-3947. 1997.

[10] García, M.J., Rebolledo, F. and Gotor, V., Tetrahedron, 23. 69356940. 1994.

[11] Fischer, A., Bommarius, A.S., Dranz, K. and Wandrey, C., Biocatalysis, 8. 289-307. 1994.

[12] Tang, P. and Ye, F., "Boron-mediated amidation of carboxylic acids." US Patent 638427.

[13] Tang, P., Org. Synth., 81. 262. 2005.

[14] Mylavarapu, R.K., Kondaiah, G.C.M., Kolla, N., Veeramalla, R., Koilkonda, P., Bhattacharya, A. and Bandichhor, R., Org. Process Res. Dev., 11. 1065-1068. 2007.

[15] Ishihara, K., Ohara, S. and Yamamoto, H., Org. Synth., Coll. Vol. $X, 80.2004$.

[16] Trapani, G., Rheo, A. and Latrofa, A., Synthesis,1013-1014.1983.

[17] Peneux, L., Loupy, A. and Volatron, F., Tetrahedron, 58. 21552162. 2002. 